\title{
Chronic migraine and medication-overuse headache
}

Eric J. Eross, D.O.

\begin{abstract}
Many medications that people take for migraine headaches can actually make headaches worse, if the medications are taken too frequently. When this occurs, the resulting problem is called "medication-overuse headaches." Unfortunately, when medicationoveruse headaches occur, they can be difficult to treat. You can read more about migraine headaches and medication-overuse headaches on the next page.
\end{abstract}

\section{How can medication-overuse headaches be treated?}

In this issue of Neurology, Zeeberg and colleagues ${ }^{1}$ looked at the effect of a 2-month drug-free period in patients suffering from probable medication-overuse headache. They closely followed 337 patients seen at the Danish Headache Center with medication-overuse headache. The goal was to see the effects on headache frequency solely by eliminating the suspected culprit acute medication. Of the 337 patients who had their medications abruptly withdrawn, $64 \%$ (216 patients) were able to stay medication-free for 2 months. The researchers found that overall, $45 \%$ of the patients had improved in 2 months, $48 \%$ had no change, and only $7 \%$ had more headaches.

\section{Does the original headache type leading to probable medication-overuse headache determine response to acute medication withdrawal? \\ Of the 216 patients with medication-overuse headache, the original headache types leading to}

overtreatment included migraine $(10 \%)$, tension-type headache $(33 \%)$, both migraine and tensiontype headache $(43 \%)$, and other $(14 \%)$. Importantly, a previous diagnosis of migraine appeared to predict a better response to medication withdrawal. Patients with migraine only initially enjoyed a $67 \%$ reduction in headache frequency. This was dramatically different than those individuals who initially had tension-type headache only or another type of headache; these patients had absolutely no reduction of headache frequency 2 months after medication withdrawal. Patients who had both tension-type headache and migraine had a $37 \%$ reduction in overall headache frequency.

\section{Does it matter which medications were the potential culprits in probable medication-overuse headache?}

Of the 216 patients, $4 \%$ were overusing ergots, $6 \%$ narcotics, $20 \%$ triptans, 29\% simple analgesics (pain relievers), and $42 \%$ combination analgesics (multiple pain relievers in one tablet). The ergots and triptan drugs are considered "acute" treatments for headache because when they are effective, they shut down the headache quickly. Narcotics and analgesics are given to simply relieve the headache pain. The researchers found that the response to medication withdrawal also depended on the medication being overused. For example, patients overusing ergots and triptans showed the most im- provement whereas patients taking narcotics and analgesics were less likely to respond (only one in three improved).

\section{What is the takeaway message from the Danish study?}

Even if no other therapies are begun, eliminating pain and other acute medications is often an effective treatment in cases of probable medication-overuse headache. In particular, migraineurs and patients overusing ergots and triptans may benefit the most. Most experts agree that eliminating potential culprit medications is the cornerstone of successful treatment. Often, clinicians will use other "tricks of the trade" to improve a patient's odds for success such as starting a drug designed to prevent headaches (known as a prophylactic agent). Usually patients will also be supplied with alternative acute agents thought to be less likely to perpetuate the headache cycle.

The most important thing to remember is that while pain and other acute medications can be extremely effective for treating the occasional migraine headache, using them more than twice a week can actually lead to a problem that can be worse than the migraine headache itself.

\section{Reference}

1. Zeeberg P, Olesen J, Jensen R. Probable medication-overuse headache: the effect of a 2-month drug-free period. Neurology 2006; 66:1894-1898 


\section{NEUROLOGY Patient Page - About chronic migraine and medication-overuse headache}

\begin{abstract}
What is migraine and how common is it?

Migraine headache is a common medical condition that afflicts an estimated 28 million Americans, nearly as many who suffer from asthma, diabetes, and osteoarthritis combined. Over $80 \%$ of these migraineurs (patients suffering from migraine) state that they experience either severe or extremely severe pain during their attacks, and over half experience severe headacherelated disability. Not uncommonly the pain is on one side of the head, takes on a throbbing quality, and is made worse with routine physical activity such as going up stairs or bending over. Patients often experience nausea (with or without vomiting) as well as sensitivity to light (photophobia) and sound (phonophobia).
\end{abstract}

\section{What causes migraine to increase in frequency?}

With regard to headache frequency, experts generally divide migraine into either an episodic or chronic condition (headache occurring greater than 15 days per month for longer than 3 months). Risk factors for progressing from an episodic pattern to a chronic pattern include such things as education/socioeconomic status, obesity, baseline attack frequency, sleep apnea (airway obstruction during sleep), stressful life events, caffeine consumption, and notably the overuse of medications designed to get rid of an existing headache (known as an "acute" or "abortive" drug). These medications are also known as "symptomatic medications." Of these risk factors, experts believe that limiting a patient's exposure to acute drugs may be one of the most effective ways to prevent the progression to chronic daily headache.

\section{What is medication-overuse headache and what causes it? Medication-overuse headache, pre- viously know as "rebound head- ache," is a condition in which headaches become more and more frequent as a patient begins to use more and more acute headache medications. It's a downward spiral in the truest sense; the more medi- cations you use, the more head- aches you get, thus the inclination to use more of the culprit medica- tion, and so on. Researchers are unsure as to what causes medication-overuse headache, but most agree that migraineurs are particularly susceptible to this phenomenon.}

\section{Which medications cause medication-overuse headache and how much is too much?} Almost all symptomatic medications have the potential to cause medication-overuse headache if used too frequently. Having said that, experts believe that some medications have a higher potential than others. Studies suggest that butalbital-containing drugs (i.e., Fiorinal and Fioricet), narcotics, acetaminophen (Tylenol), ergot compounds (e.g., Cafergot), nonsteroidal anti-inflammatory drugs (NSAIDs), and the migraine-specific drugs known as the triptans may be the most common culprits. Dihydroergotamine (Migranal Nasal Spray), antiemetics (used for both headache and nausea), COX-2 inhibitors (e.g., Celebrex), and straightforward aspirin are thought to have a very low risk. Generally speaking, experts advise patients to avoid taking symptomatic medications more than two days per week. We do not know how long it takes to develop this condition, but some suggest it can occur within a few short months.

\section{What should I do if I think I might have medication- overuse headache?}

If you are taking one of the medications listed above, or any other pain medication more than twice a week for headache, you are at risk for developing medication-overuse headache. If you think you have developed medication-overuse headache, you should talk to your doctor about other options for treating your headache problem. If your headaches have become severe enough that you feel you need pain medication more than twice a week, you should also talk with your doctor about the best treatment strategy for you. Trying to treat the problem on your own, even with frequent use of over-thecounter pain medications, could actually make the problem worse and lead to medication-overuse headache.

\section{For more information \\ The National Headache Foundation www.headaches.org}

American Academy of Neurology Foundation www.thebrainmatters.org 


\section{Neurology}

\section{Chronic migraine and medication-overuse headache \\ Eric J. Eross \\ Neurology 2006;66;E43-E44 \\ DOI 10.1212/01.wnl.0000227588.24559.89}

This information is current as of June 26, 2006

\section{Updated Information \& Services}

References

Permissions \& Licensing

Reprints including high resolution figures, can be found at: http://n.neurology.org/content/66/12/E43.full

This article cites 1 articles, 1 of which you can access for free at: http://n.neurology.org/content/66/12/E43.full\#ref-list-1

Information about reproducing this article in parts (figures,tables) or in its entirety can be found online at:

http://www.neurology.org/about/about_the_journal\#permissions

Information about ordering reprints can be found online: http://n.neurology.org/subscribers/advertise

Neurology ${ }^{\circledR}$ is the official journal of the American Academy of Neurology. Published continuously since 1951, it is now a weekly with 48 issues per year. Copyright . All rights reserved. Print ISSN: 0028-3878. Online ISSN: 1526-632X.

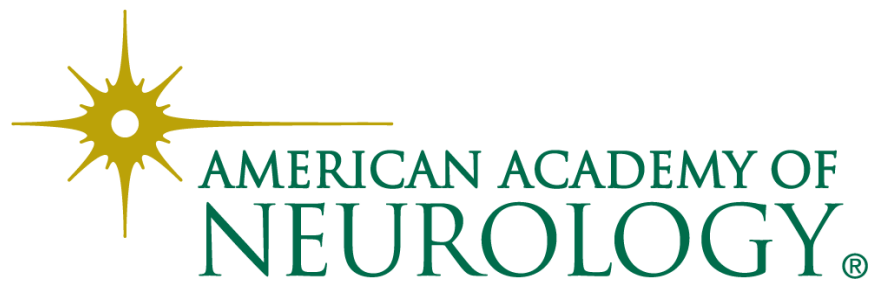

